\title{
RELACIÓN ENTRE ACTITUDES SEXISTAS Y VARIABLES EMOCIONALES POSITIVAS Y NEGATIVAS
}

\author{
MAITE GARAIGORDOBIL \\ CARMEN MAGANTO \\ Universidad del País Vasco
}

Recibido 9-12-2014

Aceptado 12-04-2015

\section{Resumen}

La investigación tuvo dos objetivos: (1) analizar las relaciones existentes entre sexismo (SH-hostil, SB-benevolente, SA-ambivalente) con emociones positivas y negativas; y (2) identificar variables predictoras del SH y SB. La muestra está constituida por 941 estudiantes de 14 a 25 años. Los resultados evidenciaron correlaciones positivas del SH y del SA con ansiedad estado/rasgo, ira-estado/rasgo, con el índice de expresión de la ira; y correlaciones negativas con sentimientos de felicidad y autoestima. El SB no correlacionó con ninguna variable. Las variables predictoras del SH fueron: pocos sentimientos de felicidad, bajo nivel de depresión-estado y de ansiedad-rasgo, alto nivel de ira-estado y de expresión de la ira. Pocos sentimientos de felicidad, bajo nivel de depresión-estado, y alto de ira-estado fueron predictoras del SB. Se discute el efecto de las emociones positivas en las actitudes sexistas.

Palabras clave: Sexismo, autoestima, felicidad, ansiedad, depresión, ira, desarrollo emocional.

\begin{abstract}
The investigation had two goals: (1) to analyze the relations between sexism (HS-hostile, BS-benevolent, AS-ambivalent) and positive and negative emotions; and (2) to identify predictor variables of HS and BS. The sample comprised 941 students from 14 to 25 years old. The results revealed positive correlations of HS and AS with state/trait anxiety, state/trait anger, and the anger expression index; and negative correlations
\end{abstract}


with feelings of happiness and self-esteem. BS was not correlated with any variable. The predictor variables of HS were: few feelings of happiness, low level of state-depression and of trait-anxiety, high level of state-anger and anger expression. Few feelings of happiness, low level of state-depression, and high level of state-anger were predictors of BS. The effect of positive emotions on sexist attitudes is discussed.

Key-words: Sexism, self-esteem, happiness, anxiety, depression, anger, emotional development. 


\section{Introducción}

El sexismo se define como una actitud discriminatoria dirigida a las personas en virtud de su pertenencia a un determinado sexo biológico, en función del cual se asumen diferentes características y conductas. Es incuestionable que las mujeres han hecho grandes progresos en disminuir la desigualdad de género, no obstante, los resultados de los estudios actuales confirman que la persistencia de la discriminación en función del género continúa siendo un problema de la sociedad contemporánea. En este sentido, algunos estudios ${ }^{1}$, muestran que las personas tienden a creer que la discriminación de género ya no es un problema de las sociedades contemporáneas; y esto podría explicarse porque las expresiones sexistas se han hecho más evasivas y difíciles de reconocer.

Desde una perspectiva psicosocial de género, el sexismo es considerado una de las principales creencias que mantienen las desigualdades entre sexos ${ }^{2}$. Una aportación importante para la comprensión del sexismo la han realizado Glick y Fiske ${ }^{3}$ identificando el Sexismo Ambivalente, resultado de la combinación de dos tipos de sexismo con cargas afectivas antagónicas. El Sexismo Hostil comparte con el sexismo más tradicional su carga afectiva negativa y supone asumir una visión estereotipada y negativa de la mujer como ser inferior. Junto al hostil convive el Sexismo Benevolente de tono afectivo positivo, basado en una ideología tradicional, que idealiza a las mujeres como esposas, madres y objetos románticos, pero sigue siendo sexista ya que las relega a

1. BARreto, Manuela; Ellemers, Naomi; Cihangir, Sezgin y Stroebe, Katherine: «The self-fulfilling effects of contemporary sexism: How it affects women's well-being and behaviour». En Manuela Barreto, Michelle Ryan y Michael Schmitt (dirs.). The glass ceiling in the 21st century: Understanding barriers to gender equality. Washington, American Psychological Association, 2009, pp. 99-123.

2. Moya, Miguel. "Actitudes sexistas y nuevas formas de sexismo». En Ester Barberá y Isabel Martínez-Benlloch (dirs.). Psicología y Género. Madrid, Pearson, 2004, pp. 271-294.

3. GLICK, Peter y FISKE, Susan. «The Ambivalent sexism inventory: differentiating hostile and benevolent sexism». Journal of Personality and Social Psychology 70 (1996), pp. 491-512. 
estos roles, considerando a la mujer como una persona débil a la que los hombres deben proteger.

Desde la Teoría del Sexismo Ambivalente, Glick y Fiske ${ }^{4}$, sugieren que tanto el Sexismo Hostil como el Sexismo Benevolente tienen sus raíces en las condiciones biológicas y sociales que son comunes a todos los grupos humanos; y giran en consecuencia en torno al poder social, la identidad de género y la sexualidad, articulándose en torno a 3 componentes comunes: El paternalismo, la diferenciación de género y la heterosexualidad. Cada componente refleja una serie de creencias en las que la ambivalencia hacia las mujeres es inherente, ya que presenta un componente hostil y otro benévolo.

El paternalismo se define como la forma en la que un padre se comporta con sus hijos e hijas, por un lado les aporta afecto y protección y, por otro les da órdenes. Esta concepción está relacionada con la visión ambivalente del sexismo porque incluye dos dimensiones. El paternalismo dominador, que desencadena el Sexismo Hostil, donde se asienta la estructura del patriarcado que legitima la superioridad de la figura masculina, y ve a las mujeres como seres incapaces, incompetentes, y peligrosas porque intentan arrebatar el poder a los hombres. El paternalismo protector, desencadena el Sexismo Benevolente, y los hombres aplican a las mujeres que desempeñan roles tradicionales (esposas, madres y objetos románticos), ya que las consideran como criaturas débiles y frágiles, a las que hay que colocar en un pedestal y protegerlas. Así, las mujeres tienen que ser amadas, acariciadas y protegidas, ya que su debilidad requiere que los hombres cumplan con su papel protector y de sustento económico.

En la diferenciación de género también se expresan las dos caras del sexismo. La diferenciación de género competitiva se presenta como una justificación sobre el poder estructural masculino, ya que considera que solamente los hombres poseen los rasgos necesarios para poseer el poder y gobernar las instituciones socio-económicas y políticas. A su vez, las mujeres al ser diferentes de los hombres, no cuentan con las características, ni con la capacidad necesarias para poder gobernar y, por tanto, su ámbito de actuación quedaría limitado a la familia y al hogar. La diferenciación de género complementaria asume que las «características» de las mujeres son «complementarias» a las de los hombres. Para el sexista benevolente, las características de las mujeres complementan las características de los hombres, mientras que para el hostil, determinadas características de las mujeres como la sensibilidad, las colocan en un plano inferior y las hacen incompetentes para ejercer el poder.

4. GLICK, Peter, y FISKE, Susan. Op. cit.

Feminismo/s 25, junio 2015, pp. 35-54 
Finalmente, desde esta teoría sitúan en la heterosexualidad uno de los más poderosos orígenes de las actitudes ambivalentes de los hombres hacia las mujeres. Igual que los anteriores componentes tiene dos vertientes: la intimidad heterosexual y la hostilidad heterosexual. La motivación sexual de los hombres hacia las mujeres puede estar unida a un deseo de proximidad (intimidad heterosexual), lo que alimenta el Sexismo Benevolente. Sin embargo, para algunos hombres la atracción sexual hacia las mujeres puede ser inseparable del deseo de dominarlas, lo que se denominaría hostilidad heterosexual, y consideran que las mujeres debido a su poder sexual, son peligrosas y manipuladoras, lo que alimenta el Sexismo Hostil.

Las actitudes sexistas impiden considerar a la mujer en situación de igualdad, aún desempeñando similar responsabilidad laboral ${ }^{5}$. La investigación sobre sexismo y emociones ha tenido un desarrollo desigual en razón de las emociones estudiadas, pero en general su investigación ha sido escasa. Los estudios sobre sexismo y atribución emocional indican que los hombres sexistas hostiles atribuyen menos emociones positivas a las mujeres, y los hombres sexistas benevolentes atribuyen más emociones positivas. Así mismo, el sexismo hostil atribuye menos emociones favorables a las mujeres de carrera, mientras que el sexismo benevolente atribuye emociones más favorables a las amas de casa ${ }^{6}$. Con esta fundamentación, en el presente estudio se analiza la relación del sexismo con emociones positivas (felicidad, autoestima) y negativas (ansiedad, depresión, ira).

Las investigaciones que han analizado la relación entre sexismo y autoestima, en general muestran que las personas sexistas tienen inferior autoestima, al compararlas con las personas con bajos niveles de sexismo. El estudio de Angell ${ }^{7}$ encontró que los que eran menos sexistas tenían mayor autoestima, y el trabajo de Lameiras y Rodríguez ${ }^{8}$ halló que las mujeres sexistas benevolentes y los hombres sexistas hostiles tenían baja autoestima. Uno de los componentes esenciales de la autoestima en la adolescencia es el propio cuerpo, utilizado como el interfaz de las relaciones interpersonales con

5. Walton, Robert y Politano, Michael. «Gender-Related Perceptions and Stress, Anxiety, and Depression on the Flight Deck». Aviation Psychology and Applied Human Factors 4 (2014), pp. 67-73.

6. GAUNT, Ruth. «Ambivalent sexism and the attribution of emotions to men and women». Revue Internationale de Psychologie Sociale 26 (2013), pp. 29-54.

7. ANGELL, Marie Louise. «Race, sex roles, and sexuality». Dissertation Abstracts International: Section B: The Sciences and Engineering 65(8-B) (2005), p. 4335.

8. LAMEIRAS, María y RODRíGUEZ, Yolanda. «Evaluación del sexismo ambivalente en estudiantes gallegos/as». Acción Psicológica 2 (2003), pp.131-136. 
los iguales ${ }^{9}$. Los estudios demuestran que la autoestima en las mujeres está muy vinculada a su apariencia física ${ }^{10}$, mientras que en los hombres está más relacionada con otras variables como prestigio, poder o competitividad. Los problemas de baja autoestima e imagen corporal son las variables de mayor correlación entre sí y las que confirman con más consistencia su relación negativa con sexismo ${ }^{1112}$.

Los estudios sobre sexismo y felicidad/bienestar psicológico, indican que la aceptación del Sexismo Benevolente proporciona satisfacción vital y mayor felicidad en hombres y mujeres. En los varones, el Sexismo Benevolente correlaciona directamente con la satisfacción vital, mientras que para las mujeres los efectos ocurren de forma indirecta. Las mujeres que aceptan y se identifican con el posicionamiento ideológico del Sexismo Benevolente por parte de los varones, es decir, se adhieren emocionalmente a unas relaciones de género basadas en la desigualdad, pero que propician adoración, adulación y protección, son las que informan de niveles más altos de satisfacción vital y felicidad ${ }^{13}$. Estas mismas correlaciones positivas entre Sexismo Benevolente y felicidad, en ambos sexos, han sido informadas por Napier, Thorisdottir, y Jost $^{14}$. Sin embargo, también hay evidencia de la relación negativa con bienestar psicológico ${ }^{15}$.

No hay estudios que hayan correlacionado sexismo y emociones negativas, aunque en una línea de investigación complementaria, son múltiples los estudios que verifican que altos niveles de discriminación percibida

9. Morin, Alexandre; MAÏANO, Christophe; MARSH, Herbert; JANOSZ, Michel y NAGENGAST, Nagengast. "The longitudinal interplay of adolescents' self-esteem and body image: a conditional autoregressive latent trajectory analysis. Multivariate Behavioral Research 46 (2011), pp. 157-201.

10. SMOLAK, Linda y MuRnEN, Sarah. «Drive for leanness: Assessment and relationship to gender, gender role and objectification». Body Image 5 (2008), pp. 251-260.

11. Barreto, Manuela; Ellemers, Naomi; Cihangir, Sezgin y Stroebe, Katherine. Op. cit.

12. Sheferd, Melisa; Erchull, Mindy; Rosner, Aryn; Taubenberger, Leslie; QueEn, Emily y MCKEE, Jenna. «I'll Get That for You: The Relationship Between Benevolent Sexism and Body Self-Perceptions». Sex Roles 64 (2011), pp. 1-8.

13. HAmmond, Matthew y Sibley, Chris. «Why are benevolent sexists happier?» Sex Roles 65 (2011), pp. 332-343.

14. NAPIER, Jaime; THORISDOTTIR, Hulda y JOST, John. «The joy of sexism? A multinational investigation of hostile and benevolent justifications for gender inequality and their relations to subjective wellbeing». Sex Roles 62 (2010), pp. 405-419.

15. FISCHER, Ann y BOLTON, Kenna. «Testing a model of women's personal sense of justice, control, well-being and distress in the context of sexist discrimination». Psychology of Women Quarterly 34 (2010), pp. 297-310. 
correlacionan con altos niveles de alteraciones emocionales y de malestar psicológico $^{1617}$. La discriminación es una experiencia estresante que disminuye la autoestima personal ${ }^{18}$, ocasionando mayor nivel de depresión y ansiedad en las personas que han experimentado eventos sexistas de discriminación ${ }^{19}$. Fischer y Bolton ${ }^{20}$ encontraron correlaciones positivas entre discriminación sexista y depresión (.37) y ansiedad (.29). En concreto, se ha demostrado que a mayor sentimiento de injusticia por discriminación sexual percibida mayor nivel de ansiedad y depresión ${ }^{2122}$.

Apenas existen estudios que hayan explorado las relaciones entre sexismo e ira. Cabe destacar el estudio de Spence, Losoff y Robbins ${ }^{23}$ con universitarios en el que encontraron correlaciones positivas entre ira y sexismo en ambos sexos. De la relación de la ira con otros aspectos muy relacionados con el sexismo como son las actitudes favorables hacia la violación, el estudio de Sierra, Santos-Iglesias, Gutiérrez-Quintanilla, Bermúdez y Buela-Casal ${ }^{24}$ halló que tanto la ira-estado, como la ira-rasgo correlacionan positiva y significativamente con estas actitudes. Además, estudios recientes ${ }^{25}$ han evidenciado correlaciones positivas entre Sexismo Hostil y Sexismo Benevolente con dife-

16. MORADI, Bonnie y HASAN, Nadia. «Arab American persons' reported experiences of discrimination and mental health: The mediating role of personal control». Journal of Counseling Psychology 51 (2004), pp. 418-428.

17. SMITH, Nathan e INGRAM, Kathleen. «Workplace heterosexism and adjustment among lesbian, gay, and bisexual individuals: The role of unsupportive social interactions». Journal of Counseling Psychology 51 (2004), pp. 57-67.

18. CASSIDY, Clare; O'Connor, Rory; Howe, Christine y Warden, David. «Perceived discrimination and psychological distress: The role of personal and ethnic self-esteem». Journal of Counseling Psychology 51 (2004), pp. 329-339.

19. MORADI, Bonnie y SUBICH, Linda Mezydlo. «An examining the moderating role of sefsteem in the link between experiences of perceived sexist events to psychological distrees for African American women». The Counseling Psychologist 51 (2004), pp. 50-56.

20. FISCHER, Ann y BOLTON, Kenna. «Perceived discrimination and women's psychological distress: The roles of collective and personal self-esteem». Journal of Counseling Psychology 54 (2007), pp. 154-164.

21. MORADI, Bonnie y HASAN, Nadia. Op. cit.

22. MORADI, Bonnie y SUBICH, Linda Mezydlo. Op. cit.

23. SPENCE, Janet; LOSOFF, Michael y ROBBINS, Ann. «Sexually aggressive tactics in sating relationships: Personality and attitudinal correlates». Journal of Social and Clinical Psychology 10 (1991), pp. 289-304.

24. Sierra, Juan Carlos; Santos-Iglesias, Pablo; GutiérReZ-Quintanilla, Ricardo; BerMÚDEZ, María Paz y BUELA-CASAL, Gualberto. «Factors associated with rape-supportive attitudes: Sociodemographic variables, aggressive personality, and sexist attitudes». Spanish Journal of Psychology 13 (2010), pp. 202-209.

25. ROJAS-SOLÍs, José Luis y CARPINTERO, Eugenio. «Sexismo y agresiones físicas, sexuales y verbales-emocionales, en relaciones de noviazgo de estudiantes universitarios». Electronic Journal of Research in Educational Psychology 9 (2011), pp. 541-564.

Feminismo/s 25, junio 2015, pp. 35-54 
rentes tipos de conductas agresivas (sexuales, físicas, verbales-emocionales), encontrando los índices de correlación más altos con agresión sexual.

Estos resultados permiten sugerir la necesidad de mayor nivel de investigación para clarificar el campo del área de las relaciones entre sexismo y emociones positivas y negativas. Con esta contextualización, el estudio tuvo dos objetivos: 1) analizar las relaciones existentes entre Sexismo Hostil, Sexismo Benevolente y Sexismo Ambivalente con emociones positivas (sentimientos de felicidad y autoestima) y con emociones negativas (ansiedad, depresión, e ira); y 2) identificar variables predictoras de Sexismo Hostil y del Sexismo Benevolente. Con estos objetivos, y teniendo en cuenta los estudios previos, la investigación se plantea dos hipótesis: 1) El sexismo tendrá relaciones negativas con sentimientos de felicidad y autoestima, así como relaciones positivas con ansiedad, depresión e ira; y 2) Alto nivel de emociones negativas y bajo nivel de emociones positivas serán variables predictoras de alto nivel de sexismo.

\section{Método}

\subsection{Participantes}

La muestra está constituida por 941 participantes de 14 a 25 años, 465 hombres $(49,4 \%)$ y 476 mujeres $(50,6 \%)$ del País Vasco. Los participantes se distribuyen en dos rangos de edad, 471 adolescentes de 14 a 18 años $(50,1 \%)$ y 470 jóvenes de 19 a 25 años (49,9\%). La distribución por sexos y niveles educativos puede observarse en la Tabla 1. La muestra es una muestra representativa de los estudiantes de ESO, Bachiller y Universidad del País Vasco. Según la última encuesta presentada por el Instituto Vasco de Estadística (eustat.es), la población de estudiantes de ESO y Bachiller en el País Vasco es 101.757 $(72.272+29.485)$, y los que cursan estudios universitarios 64.127. Utilizando un nivel de confianza de .95 , con un error muestral de .05, para una varianza poblacional de .50, la muestra representativa es de 766. La muestra fue seleccionada mediante una técnica de muestreo aleatorio simple a partir de la lista de centros educativos del País Vasco (ESO y Bachiller), incluyendo estudiantes universitarios de diversas facultades: Derecho, Psicopedagogía, Psicología, Enfermería, Ingeniería Industrial y Educación Física. 
Tabla 1. Frecuencias y porcentajes de varones y mujeres en cada nivel educativo

\begin{tabular}{|l|c|c|c|c|c|}
\hline \multicolumn{7}{|c|}{ Niveles Educativos } \\
\hline & ESO & Bachiller & $\begin{array}{c}\text { Universidad } \\
(\text { cursos 1-2) }\end{array}$ & $\begin{array}{c}\text { Universidad } \\
(\text { cursos 3-5) }\end{array}$ & Total \\
\hline Varón & $131(13.9 \%)$ & $118(12.5 \%)$ & $111(11.8 \%)$ & $105(11,2 \%)$ & $465(49,4 \%)$ \\
\hline Mujer & $114(12.1 \%)$ & $108(11.5 \%)$ & $118(12.5 \%)$ & $136(14.5 \%)$ & $476(50,6 \%)$ \\
\hline Total & $245(26.0 \%)$ & $226(24.0 \%)$ & $229(24,3 \%)$ & $241(25.6 \%)$ & $941(100 \%)$ \\
\hline
\end{tabular}

Notas: $\mathrm{ESO}=$ Educación Secundaria Obligatoria.

\subsection{Instrumentos de Evaluación}

Con la finalidad de medir las variables objeto de estudio se utilizaron seis instrumentos de evaluación con garantías psicométricas de fiabilidad y validez.

ASI. Inventario de sexismo ambivalente ${ }^{2627}$. El inventario consta de 22 frases, a las que se responde con una escala que va de 0 (totalmente en desacuerdo) a 5 (totalmente de acuerdo). La prueba mide el Sexismo Ambivalente (SA) compuesto de dos dimensiones: Sexismo Hostil ( $\mathrm{SH}$, que implica un estereotipo de la mujer como ser inferior) y Sexismo Benevolente (SB, entendido como un conjunto de actitudes sexistas hacia las mujeres en cuanto que las considera positivamente, pero de forma estereotipada y limitada a sus roles de madre, esposa y objeto romántico). Los estudios de fiabilidad han evidenciado una consistencia interna (alfa de Cronbach) alta para SA $(\alpha=.90)$ y sus subescalas ( $\mathrm{SH} \alpha=.89$; SB $\alpha=.86$ ). Los estudios de validez del ASI han mostrado correlaciones significativas del SA con la Escala de Ideología de Género, así como con la escala de Neosexismo, de mayor magnitud con la subescala de SH. La consistencia interna obtenida con la muestra del presente estudio fue alta $(\alpha=.91)$.

RSE. Escala de autoestima ${ }^{28}$. Esta escala evalúa la autoestima general con 10 afirmaciones que aluden a sentimientos globales de autovaloración, de satisfacción con uno mismo. La persona debe leer las afirmaciones e informar en qué medida pueden ser aplicadas a sí misma, haciendo la valoración

26. GLICK, Peter y FISKE, Susan. Op. cit.

27. MoYa, Miguel; EXPÓSITO, Francisca y PADILla, José Luis. «Revisión de las propiedades psicométricas de las versiones larga y reducida de la escala sobre ideología de género». International Journal of Clinical and Health Psychology 6 (2006), pp. 709-727.

28. RosenberG, Morris. Society and the adolescent self-image. Princeton, NJ, Princeton University Press, 1965. 
sobre una escala con 4 categorías de respuesta (de muy de acuerdo a muy en desacuerdo). La fiabilidad ha sido ampliamente documentada en la literatura. McCarthy y Hoge ${ }^{29}$ han informado de coeficientes de consistencia (alfa de Cronbach) que se sitúan entre .74 y .77 , y de fiabilidad test-retest de .63 (intervalo de 7 meses) y de .85 (intervalo de 2 semanas). La validez como medida unidimensional de la autoestima ha sido también comprobada ${ }^{30}$. La consistencia interna obtenida con la muestra del presente estudio fue adecuada $(\alpha=.83)$.

OHQ. El cuestionario de felicidad de Oxford ${ }^{31}$. Esta escala mide la felicidad de carácter general de cada individuo, el bienestar psicológico. Se configura con 29 ítems y la persona expresa su grado de acuerdo con una escala de 1 a $6(1=$ totalmente en desacuerdo, $6=$ totalmente de acuerdo). Los estudios realizados de esta escala, con una muestra de personas entre 13 y 68 años, verifican una buena fiabilidad $(\alpha=.91)$. La validez del constructo del OHQ fue evaluada con asociaciones de diferentes medidas de diferencias individuales de rasgos y variables cognitivas, confirmándose su validez. La adaptación española evidenció coeficientes adecuados de fiabilidad y validez ${ }^{32}$. La consistencia interna obtenida con la muestra del presente estudio fue adecuada $(\alpha=.86)$.

STAI. Cuestionario de Ansiedad Estado-Rasgo ${ }^{33}$. El STAI evalúa la ansiedad como estado (AE) (condición emocional transitoria que puede variar con el tiempo y fluctuar en intensidad) y la ansiedad como rasgo (AR) (propensión ansiosa relativamente estable, que permanece latente hasta que es activada por algún estímulo situacional). Consta de 40 elementos, a los que se responde con una escala de nada a mucho (AE) y de casi nunca a casi siempre (AR). Los análisis psicométricos de la versión original han tenido buenos resultados. En la adaptación española, se obtuvo una buena consistencia interna (AE: entre .90 y .93; AR: entre .84 y .87). Estos índices se asemejan a los obtenidos en los estudios originales (entre .83 y .92). La validez concurrente obtenida en

29. MCCARTHY, John y Hoge, Dean. "Analysis of age effects in longitudinal study of adolescent self-esteem». Developmental Psychology 18 (1982), pp. 372-379.

30. SILBER, Earl y TIPPETT, Jean. «Self-esteem: clinical assessment and measurement validation». Psychological Reports 16 (1965), pp. 1017-1071

31. Hills, Peter y ARGYLE, Michael. «The Oxford Happiness Questionnaire: a compact scale for the measurement of psychological well-being» Personality and Individual Differences 33 (2002), pp. 1073-1082

32. GARAIGORDOBIL, Maite y DURÁ, Ainhoa. «Neosexismo en adolescentes de 14 a 17 años: Relaciones con autoconcepto-autoestima, personalidad, psicopatología, problemas de conducta, y habilidades sociales». Clínica y Salud 17 (2006), pp. 127-149.

33. SPIELBERGER, Charles; Gorsuch, Richard y LuSHENE, Robert Edward. STAI Cuestionario de Ansiedad Estado-Rasgo. Manual. Madrid, TEA, 1970. 
estos estudios (relacionando el STAI con otras medidas de ansiedad) oscila entre .58 y .76 en muestras de universitarios varones, y entre .52 y .80 con mujeres universitarias. En la adaptación española también se han obtenido índices adecuados, por ejemplo, se ha encontrado una elevada correlación (.88 en situación normal y .82 en situación experimental) entre el STAI-AE y el Eight State Questionnaire (ESQ), aunque esta relación se redujo bastante en la escala AR (.29 y .26 respectivamente). La consistencia interna obtenida con la muestra del presente estudio fue adecuada $(\alpha=.70)$.

IDER. Inventario de Depresión Estado-Rasgo ${ }^{34}$. El IDER tiene como objetivo identificar el grado de afectación (DE. Depresión-Estado) y la frecuencia de ocurrencia (DR. Depresión-Rasgo) del componente afectivo de la depresión. Contiene 20 afirmaciones, de las cuales 10 son para identificar DE y 10 para DR. Los estudios psicométricos han evidenciado coeficientes de fiabilidad alfa de Cronbach en DE que oscilan entre.89 y.92, y en DR entre.81 y.86. Estudios de validación han confirmado correlaciones con el Inventario de Depresión de Beck BDI que oscilan entre. 51 y.64. La consistencia interna obtenida con la muestra del presente estudio fue adecuada $(\alpha=.88)$.

STAXI-2. Inventario de Expresión de Ira Estado-Rasgo ${ }^{35}$. El STAXI-2 mide la experiencia y la expresión de la ira (IE = ira estado; IR = ira rasgo; IEI = índice de expresión de la ira). La prueba se compone de 49 ítems que permiten obtener un Índice de Expresión de la Ira (IEI) que representa una medida global de la expresión y control de la ira. IE se explora con 15 afirmaciones y la persona informa si tiene los sentimientos que se indican en ese momento, respondiendo con una escala de nada a mucho. IR contiene 10 afirmaciones con las que la persona informa si habitualmente tiene los sentimientos que se indican, respondiendo con una escala de casi nunca a casi siempre. El IEI incluye 24 afirmaciones y la persona informa de su forma de reaccionar cuando se enfada, respondiendo con una escala de casi nunca a casi siempre. Los resultados psicométricos han evidenciado buenos niveles de fiabilidad (test-retest: IR.:71; IEI =.62) y consistencia interna (alfa de Cronbach: IR:.82; IEI =.69). Estudios de validación han puesto de relieve la validez de constructo, las correlaciones encontradas de IR con el índice de hostilidad del inventario de Buss-Durkee fueron de .66 y .71 para mujeres y varones universitarios, y de .73 y .66 con mujeres y hombres reclutas de la armada.

34. Spielberger, Charles; Buela-CASAL, Gualberto y AGUdElo, Diana. «IDER: Inventario de Depresión estado-rasgo». Madrid, TEA, 2008.

35. Miguel-Tobal, Juan José; Casado, María Isabel; Cano-Vindel, Antonio y SpielberGER, Charles. Inventario de Expresión de Ira Estado/Rasgo. Madrid, TEA, 2001. 
La consistencia interna obtenida con la muestra del presente estudio fue adecuada $(\alpha=.86)$.

\subsection{Procedimiento}

En primer lugar, se llevó a cabo una entrevista con los directores-as de los centros educativos para exponer la investigación y solicitar su colaboración. Posteriormente, se celebró una reunión con los padres de los participantes preuniversitarios para explicarles el estudio y solicitar el consentimiento informado, autorizando a sus hijos-hijas a participar en la investigación. Los cuestionarios fueron administrados de forma colectiva, voluntaria y anónima en el aula. A los participantes se les indicó que cumplimentaran los datos de identificación (género, edad...) y las instrucciones se leyeron en voz alta. Los evaluadores, licenciados en psicología, estuvieron presentes durante la administración de las pruebas para proporcionar ayuda. El tiempo medio de aplicación fue de 60 minutos. El estudio cumplió los valores éticos requeridos en la investigación con seres humanos habiendo sido evaluado favorablemente por la Comisión de Ética de la Universidad del País Vasco (UPV/EHU).

\subsection{Análisis de datos}

De forma preliminar, con la finalidad de analizar si existen diferencias en el conjunto de las variables objeto de estudio en esta investigación, ya que podrían condicionar los subsiguientes análisis, se realizó un análisis de varianza multivariante en función del género y la edad. Teniendo en cuenta los resultados del MANOVA, y con la finalidad de analizar las relaciones existentes entre sexismo y emociones, se llevaron a cabo correlaciones parciales, controlando el efecto del sexo y la edad. Finalmente, con el objetivo de identificar variables predictoras del sexismo hostil y benevolente se realizó un análisis de regresión lineal múltiple.

\section{Resultados}

\subsection{Relaciones entre sexismo y emociones (felicidad, autoestima, ansiedad, depresión e ira)}

Los resultados del MANOVA para el conjunto de las variables, Wilks $\Lambda=.732$, $F(12,920)=21.30, p<.001$, muestran diferencias de género significativas, siendo el tamaño del efecto medio $\left(\chi^{2}=.268 ; r=.51\right)$, y también se evidencian diferencias en función de la edad, Wilks $\Lambda=.844, F(12,920)=10.72, p<.001$, siendo el tamaño del efecto medio-bajo $\left(\chi^{2}=.156 ; r=.34\right)$. Los resultados 
confirmaron puntuaciones superiores en los tres tipos de sexismo en los chicos y una disminución del sexismo con la edad.

Por esta razón, con la finalidad de analizar las relaciones existentes entre sexismo y emociones, se llevaron a cabo correlaciones parciales, controlando el efecto del sexo y la edad. Los coeficientes obtenidos (ver Tabla 2) ponen de relieve correlaciones significativas $(p<.001)$ positivas del Sexismo Hostil y Sexismo Ambivalente con ansiedad estado-rasgo $(r=.11)$, ira-estado $(r=.15)$, ira-rasgo $(r=.14)$, y con el índice de expresión de la ira $(r=.18)$, así como correlaciones negativas con sentimientos de felicidad $(r=-.13)$, y autoestima $(r=-.08)$. El Sexismo Benevolente sin embargo no correlacionó con ninguna emoción. Por consiguiente, el Sexismo Hostil y Sexismo Ambivalente se relacionan con bajo nivel de sentimientos de felicidad y autoestima, así como con alto nivel de ansiedad (estado-rasgo) y de ira (experiencia y expresión), aunque estas relaciones son de baja magnitud.

Tabla 2. Coeficientes de correlación parcial, controlando el efecto del sexo y la edad, entre sexismo y emociones, intercorrelaciones y consistencia interna de las variables

\begin{tabular}{|l|c|c|c|}
\hline & $\begin{array}{c}\text { Sexismo } \\
\text { Hostil }\end{array}$ & $\begin{array}{c}\text { Sexismo } \\
\text { Benevolente }\end{array}$ & $\begin{array}{c}\text { Sexismo } \\
\text { Ambivalente }\end{array}$ \\
\hline Sexismo Benevolente & $.53^{* * *}$ & & \\
\hline Sexismo Ambivalente & $.88^{* * *}$ & $.87^{* * *}$ & \\
\hline Felicidad & $-.13^{* * *}$ & -.05 & $-.10^{* *}$ \\
\hline Autoestima & $-.08^{* * *}$ & -.04 & $-.07^{*}$ \\
\hline Ansiedad Estado & $.11^{* * *}$ & .02 & $.07^{*}$ \\
\hline Ansiedad Rasgo & $.13^{* * *}$ & .05 & $.11^{* *}$ \\
\hline Depresión Estado & .02 & .06 & .02 \\
\hline Depresión rasgo & .03 & .01 & .03 \\
\hline Ira estado & $.15^{* * *}$ & .03 & $.10^{* *}$ \\
\hline Ira rasgo & $.14^{* * *}$ & .01 & $.07^{*}$ \\
\hline Índice expresión ira & $.18^{* * *}$ & .05 & $.13^{* * *}$ \\
\hline$\alpha$ & .91 & .90 & .84 \\
\hline
\end{tabular}

Nota: $\alpha=$ coeficiente alfa de Cronbach en las variables con la muestra del estudio ${ }^{*} p<.05{ }^{* *} p<.01 \quad * * * p<.001$ 
3.2. Variables predictoras del sexismo hostil y benevolente

Con el objetivo de identificar variables predictoras del Sexismo Hostil y del Sexismo Benevolente se realizaron análisis de regresión múltiple lineal, método de pasos sucesivos, cuyos resultados se presentan en la Tabla 3. En relación a las variables predictoras del Sexismo Hostil (ver Tabla 3) cinco resultaron estadísticamente significativas: ira-estado (Beta $=.195)$, índice de expresión de la ira (Beta $=.167)$, depresión-estado (Beta $=-.168)$, sentimientos de felicidad (Beta $=-.178$ ), y ansiedad-rasgo (Beta $=-.099)$. Los coeficientes de regresión estandarizados Beta indican que las 5 variables tienen poco peso sobre la variable criterio «Sexismo Hostil» (9,3\% de la varianza). De acuerdo con esta afirmación, los porcentajes de varianza explicada (coeficientes de determinación ajustados) por cada una de tales variables predictoras fueron de baja magnitud para las 5 variables (4,3\%, 6,3\%, 7,8\%, 8,9\%, 9,3\%). Resultaron predictoras de la variable «SH»: pocos sentimientos de felicidad, bajo nivel de depresión-estado, bajo nivel de ansiedad-rasgo, alto nivel de iraestado y alto nivel de expresión de la ira.

En relación a las variables predictoras del Sexismo Benevolente (ver Tabla 3) tres resultaron estadísticamente significativas: depresión-estado $($ Beta $=-.200)$, sentimientos de felicidad (Beta $=-.142)$, e ira-estado (Beta $=.103$ ). Los coeficientes de regresión estandarizados Beta indican que estas variables tienen poco peso sobre la variable «Sexismo Benevolente» (3,5\% de la varianza). De acuerdo con esta afirmación, los porcentajes de varianza explicada (coeficientes de determinación ajustados) por cada una de tales variables predictoras fueron de baja magnitud para las 3 variables $(0,8 \%$, 2,7\%, 3,5\%). Resultaron predictoras del «Sexismo Benevolente»: pocos sentimientos de felicidad, bajo nivel de depresión-estado, y alto nivel de ira-estado. 
Tabla 3. Análisis de regresión múltiple lineal para variables predictoras de sexismo hostil y benevolente

\begin{tabular}{|l|c|c|c|c|c|c|c|c|}
\hline & $R$ & $R^{2}$ & $\Delta R^{2}$ & $B$ & Error & Constante & $\beta$ & $t$ \\
\hline $\begin{array}{l}\text { Variables predictoras } \\
\text { de sexismo hostil }\end{array}$ \\
\hline IE. Ira-Estado & .211 & .045 & .043 & .029 & .006 & 1.535 & .195 & $5.05^{* * *}$ \\
IEI. Índice Expresión Ira & .257 & .066 & .063 & .019 & .004 & 1.106 & .167 & $4.35^{* * *}$ \\
DE. Depresión-Estado & .286 & .082 & .078 & -.038 & .010 & 1.466 & -.168 & $-3.91^{* * *}$ \\
OHQ. Felicidad & .307 & .094 & .089 & -.011 & .003 & 2.881 & -.178 & $-3.75^{* * *}$ \\
AR. Ansiedad-Rasgo & .316 & .100 & .093 & -.012 & .006 & 3.329 & -.099 & $-2.07^{*}$ \\
\hline Variables predictoras & $\begin{array}{l}\mid 7 \\
\text { de sexismo benevolente }\end{array}$ \\
\hline DE. Depresión-Estado & .098 & .010 & .008 & -.042 & .009 & 2.477 & -.200 & $-4.65^{* * *}$ \\
OHQ. Felicidad & .173 & .030 & .027 & -.008 & .003 & 4.009 & -.142 & $-3.29^{* * *}$ \\
IE. Ira-Estado & .198 & .039 & .035 & .014 & .005 & 3.647 & .103 & $2.62^{* *}$ \\
\hline
\end{tabular}

\section{Discusión}

El estudio tuvo como objetivos analizar las relaciones existentes entre sexismo y emociones positivas y negativas, así como identificar variables predictoras de Sexismo Hostil y Benevolente. En primer lugar los resultados sugieren que las personas con alto nivel de Sexismo Hostil y Sexismo Ambivalente tienen también bajo nivel de sentimientos de felicidad y autoestima, así como alto nivel de ansiedad (estado-rasgo) y de ira (experiencia y expresión), es decir, bajo nivel de emociones positivas y alto nivel de emociones negativas. Por consiguiente, la hipótesis 1 se cumple parcialmente ya que no se han hallado relaciones entre Sexismo Benevolente y emociones ni positivas ni negativas.

Los resultados confirman los obtenidos en otros estudios que han encontrado relaciones inversas entre sexismo y autoestima ${ }^{36373839}$. Además, los resultados del presente estudio sugieren que las personas con alto nivel de sexismo tienen pocos sentimientos de felicidad, siendo baja felicidad un predictor del sexismo. Aunque no hay estudios que hayan analizado las relaciones entre ambos constructos, los resultados obtenidos se relacionan con los obtenidos

36. ANGELL, Marie Louise. Op. cit.

37. Barreto, Manuela; Ellemers, Naomi; Cihangir, Sezgin y Stroebe, Katherine. Op. cit. 38. LAMEIRAS, María y RODRíGUEZ Yolanda. Op. cit.

39. Sheferd, Melisa; ErChull, Mindy; Rosner, Aryn; Taubenberger, Leslie; QueEn, Emily y MCKEE, Jenna. Op. cit. 
en otros trabajos que han evidenciado relaciones negativas entre discriminación sexista y bienestar psicológico ${ }^{40}$, pero contradicen los obtenidos por algunos estudios ${ }^{4142}$ que han hallado correlaciones positivas entre Sexismo Benevolente y felicidad. Las discrepancias en los resultados podrían ser explicadas por los diferentes instrumentos de evaluación utilizados y por las diferentes edades de las muestras.

En cuanto a las relaciones del sexismo con emociones negativas, no existen estudios que hayan correlacionado sexismo con depresión, o con ansiedad. No obstante, desde una línea de investigación complementaria, se ha evidenciado una relación positiva entre percepción de discriminación sexista y ansiedad ${ }^{434445}$. En lo que a los sentimientos de ira se refiere, los resultados obtenidos son coherentes con otros estudios que han confirmado las relaciones positivas entre sexismo y sentimientos de ira ${ }^{46}$.

En segundo lugar, en este estudio se han identificado como variables predictoras del Sexismo Hostil bajo nivel de sentimientos de felicidad, de depresión-estado y de ansiedad-rasgo, así como alto nivel de ira-estado y de expresión de la ira. Pocos sentimientos de felicidad, bajo nivel de depresiónestado, y alto de ira-estado predijeron Sexismo Benevolente. Por consiguiente, la hipótesis 2 se cumple en su totalidad ya que alto nivel de emociones negativas y bajo de positivas se han identificado como predictoras de sexismo. No obstante, la varianza explicada tanto del sexismo hostil como del benevolente es baja, por lo que las predicciones con estos coeficientes deben ser interpretadas con mucha cautela.

Como limitaciones del estudio cabe destacar el uso de autoinformes, con los sesgos de deseabilidad social que implican, así como el diseño utilizado ya que no permite establecer relaciones de causalidad entre las variables. Pese a las limitaciones, los hallazgos del estudio representan una aportación válida en este ámbito de conocimiento porque evidencian el positivo papel que pueden desempeñar las emociones positivas en la disminución de las actitudes sexistas, aunque el poder explicativo de las emociones no sea muy alto.

Los resultados tienen implicaciones prácticas y sugieren implementar durante la infancia y la adolescencia, programas, estrategias, o actividades

40. FisCHeR, Ann y BOlton, Kenna. Op. cit.

41. Hammond, Matthew y Sibley, Chris. Op. cit.

42. NAPIER, Jaime; THORISDOTTIR, Hulda y JOST, John. Op. cit.

43. MORADI, Bonnie y HASAN, Nadia. Op. cit.

44. Moradi, Bonnie y Subich, Linda Mezydlo. Op. cit.

45. SMith, Nathan e INGRAm, Kathleen. Op. cit.

46. SPEnCE, Janet; Losoff, Michael y RobBins, Ann. Op. cit.

Feminismo/s 25, junio 2015, pp. 35-54 
que fomenten variables emocionales positivas. Desde la perspectiva de la psicología positiva se abre un importante ámbito de prevención de las actitudes sexistas, en concreto, de la violencia de género. La educación en valores emocionales positivos, como los sentimientos de felicidad, una adecuada autoestima, sentimientos de bienestar psicológico, etc., aportan más beneficios y fortalezas psicológicas para la prevención de dichas actitudes, teniendo en cuenta la fuerza convergente de las emociones positivas. Una educación en valores positivos arrojaría mayores niveles de bienestar personal, familiar y social, y disminuiría los prejuicios negativos que cada cultura postula en relación a las diferencias de sexo.

Sin embargo, una adecuada prevención no debe olvidar a su vez trabajar para disminuir las emociones negativas, proponiendo actividades que fomenten el control de los sentimientos de ira (relajación, parada del pensamiento, autoinstrucciones...). Así mismo, una propuesta de futuro de cara a la prevención del sexismo, debe contemplar dotar a las mujeres de estrategias de manejo de la ansiedad y depresión frente a la percepción de actitudes sexistas, a fin de aprender a identificar sus emociones negativas frente a dichas actitudes sin culpa y ansiedad, pudiendo responder a ellas con estrategias adecuadas y fortalezas psicológicas de resolución de conflictos. Todo ello, podrá ejercer un efecto beneficioso en la dirección de disminuir el sexismo. Teniendo en cuenta los discrepantes resultados obtenidos sobre las conexiones entre sexismo y ansiedad (correlaciones positivas entre ansiedad estado-rasgo, $\mathrm{SH}$ y SA, pero ausencia de correlaciones con SB), más investigación sobre estas conexiones puede ser sugerida.

Por consiguiente, de los resultados se derivan aplicaciones prácticas y sugieren que las actitudes sexistas se pueden prevenir a través de la promoción de la emocionalidad positiva. Esta promoción se puede llevar a cabo tanto en contextos educativos, mediante la implementación de programas de intervención psicoeducativa con los grupos-aula, como a través del contexto familiar, fomentando que los padres estimulen diversas emociones positivas en sus hijos e hijas, tras tomar conciencia de la importancia que estas tienen para el desarrollo infanto-juvenil, para la adaptación personal y social.

Agradecimientos: Estudio financiado por el Ministerio de Ciencia e Innovación (MICINN) (I+D+i) (FEM2009-09456), por el Departamento de Educación, Universidades e Investigación del Gobierno Vasco (Grupo Consolidado de Investigación GIC10/66-IT-318-10) y por la UFI 11/04 de la Universidad del País Vasco (UPV/EHU). 


\section{Referencias bibliográficas}

ANGELL, Marie Louise. «Race, sex roles, and sexuality». Dissertation Abstracts International: Section B: The Sciences and Engineering 65(8-B) (2005), p. 4335.

Barreto, Manuela; Ellemers, Naomi; Cihangir, Sezgin y Stroebe, Katherine. «The self-fulfilling effects of contemporary sexism: How it affects women's well-being and behaviour». En Manuela Barreto, Michelle Ryan y Michael Schmitt (dirs.), The glass ceiling in the 21st century: Understanding barriers to gender equality. Washington, American Psychological Association, 2009, pp. 99-123.

CASSIDY, Clare; O'ConNor, Rory; Howe, Christine y WARDEN, David. «Perceived discrimination and psychological distress: The role of personal and ethnic self-esteem». Journal of Counseling Psychology 51 (2004), 329-339.

FISCHER, Ann y BOLTON, Kenna. «Perceived discrimination and women's psychological distress: The roles of collective and personal self-esteem». Journal of Counseling Psychology 54 (2007), pp. 154-164.

FISCHER, Ann y BOLTON, Kenna. «Testing a model of women's personal sense of justice, control, well-being and distress in the context of sexist discrimination». Psychology of Women Quarterly 34 (2010), pp. 297-310.

GaraigordobIL, Maite y DURÁ, Ainhoa. «Neosexismo en adolescentes de 14 a 17 años: Relaciones con autoconcepto-autoestima, personalidad, psicopatología, problemas de conducta, y habilidades sociales». Clínica y Salud 17 (2006), pp. 127-149.

GAUNT, Ruth. «Ambivalent sexism and the attribution of emotions to men and women». Revue Internationale de Psychologie Sociale 26 (2013), pp. 29-54.

GLICK, Peter y FISKE, Susan. «The Ambivalent sexism inventory: differentiating hostile and benevolent sexism». Journal of Personality and Social Psychology 70 (1996), pp.491-512.

Hammond, Matthew y Sibley, Chris. «Why are benevolent sexists happier?» Sex Roles 65 (2011), pp. 332-343.

Hills, Peter y ARGYLE, Michael. «The Oxford Happiness Questionnaire: a compact scale for the measurement of psychological well-being» Personality and Individual Differences 33 (2002), pp. 1073-1082.

LAMEIRAS, María y RODRíGUEZ Yolanda. «Evaluación del sexismo ambivalente en estudiantes gallegos/as». Acción Psicológica 2 (2003), pp. 131-136.

MCCARTHY, John y HOGE, Dean. «Analysis of age effects in longitudinal study of adolescent self-esteem». Developmental Psychology 18 (1982), pp. 372-379.

Miguel-Tobal, Juan José; CASADO, María Isabel; CANO-Vindel, Antonio y SPIELBERGER, Charles. Inventario de Expresión de Ira Estado/Rasgo. Madrid, TEA, 2001. 
MORADI, Bonnie y HASAN, Nadia. «Arab American persons' reported experiences of discrimination and mental health: The mediating role of personal control». Journal of Counseling Psychology 51 (2004), pp. 418-428.

MORADI, Bonnie y SuBICH, Linda Mezydlo. «An examining the moderating role of sef-steem in the link between experiences of perceived sexist events to psychological distrees for African American women». The Counseling Psychologist 51 (2004), pp. 50-56.

Morin, Alexandre; MaÏAnO, Christophe; MARSH, Herbert; JAnosz, Michel y NAGENGAST, Nagengast. «The longitudinal interplay of adolescents' selfesteem and body image: a conditional autoregressive latent trajectory analysis. Multivariate Behavioral Research 46 (2011), pp. 157-201.

Moya, Miguel; Expósito, Francisca y PADILlA, José Luis. «Revisión de las propiedades psicométricas de las versiones larga y reducida de la escala sobre ideología de género». International Journal of Clinical and Health Psychology 6 (2006), pp. 709-727.

MoyA, Miguel. «Actitudes sexistas y nuevas formas de sexismo». En Ester Barberá y Isabel Martínez-Benlloch (dirs.), Psicología y Género. Madrid, Pearson, 2004, pp. 271-294.

NAPIER, Jaime; THORISDOTTIR, Hulda y JOST, John. «The joy of sexism? A multinational investigation of hostile and benevolent justifications for gender inequality and their relations to subjective wellbeing». Sex Roles 62 (2010), pp. 405-419.

RojAS-SOlís, José Luis y CARPINTERO, Eugenio. «Sexismo y agresiones físicas, sexuales y verbales-emocionales, en relaciones de noviazgo de estudiantes universitarios». Electronic Journal of Research in Educational Psychology 9 (2011), pp. 541-564.

RosenberG, Morris. Society and the adolescent self-image. Princeton, NJ, Princeton University Press, 1965.

Sheferd, Melisa; ErChull, Mindy; Rosner, Aryn; Taubenberger, Leslie; QueEn, Emily y MCKEE, Jenna. «I'll Get That for You: The Relationship Between Benevolent Sexism and Body Self-Perceptions». Sex Roles 64 (2011), pp. 1-8.

Sierra, Juan Carlos; SAntos-Iglesias, Pablo; GutiérReZ-Quintanilla, Ricardo; Bermúdez, María Paz y Buela-CASAL, Gualberto. «Factors associated with rape-supportive attitudes: Sociodemographic variables, aggressive personality, and sexist attitudes». Spanish Journal of Psychology 13 (2010), pp. 202-209.

SILBER, Earl y TIPPETT, Jean. «Self-esteem: clinical assessment and measurement validation». Psychological Reports 16 (1965), pp. 1017-1071.

SMITH, Nathan e INGRAM, Kathleen. «Workplace heterosexism and adjustment among lesbian, gay, and bisexual individuals: The role of unsupportive social interactions». Journal of Counseling Psychology 51 (2004), pp. 57-67. 
SMOLAK, Linda y MuRnEN, Sarah. «Drive for leanness: Assessment and relationship to gender, gender role and objectification». Body Image 5 (2008), pp. 251-260.

SPENCE, Janet; LOSOFF, Michael y RobBins, Ann. «Sexually aggressive tactics in sating relationships: Personality and attitudinal correlates». Journal of Social and Clinical Psychology 10 (1991), pp. 289-304.

SPIElberger, Charles; Buela-CASAl, Gualberto y AGudelo, Diana. «IDER: Inventario de Depresión estado-rasgo». Madrid, TEA, 2008.

SPIElBERGER, Charles; GORSuCH, Richard y LuSHENE, Robert Edward. STAI Cuestionario de Ansiedad Estado-Rasgo. Manual. Madrid, TEA, 1970.

Walton, Robert y Politano, Michael. «Gender-Related Perceptions and Stress, Anxiety, and Depression on the Flight Deck». Aviation Psychology and Applied Human Factors 4 (2014), pp. 67-73. 